\title{
Available Bandwidth Measurement Using Poisson Probing on the Internet
}

\author{
Xiaojun Hei and Danny H.K. Tsang \\ Dept. of Electrical \& Electronic Engineering \\ Hong Kong University of Sci. \& Tech. \\ Clear Water Bay, Hong Kong \\ \{heixj, eetsang\}@ee.ust.hk
}

\author{
Brahim Bensaou \\ Dept. of Computer Science \\ Hong Kong University of Sci. \& Tech. \\ Clear Water Bay, Hong Kong \\ brahim@cs.ust.hk
}

\begin{abstract}
In this paper, we investigated a non-intrusive probing methodology for available bandwidth measurement based on the analysis of the departure process of an active Poisson probing stream. Unlike the self-congestion based available bandwidth measurement, non intrusive techniques are meant to infer the available bandwidth along a path without congesting the path. We propose to probe the end-to-end path using small size packets with exponentially distributed time between consecutive probing packets. Of particular interest to our investigations, is the Squared Coefficient of Variation (SCV) of the interdeparture process of the probing stream. The Internet is modelled as single server queue with two concurrent streams, the probing traffic stream and the cross traffic, we rely on the results on $M_{1}+M_{2} / G I_{i} / 1$ queueing system and a heavy traffic approximation model to analyze the departure process of the probing stream. Thus, in a real measurement system, given the measured SCV of the probing stream, inverting the approximation helps inferring the load of the cross traffic on an end-to-end path.
\end{abstract}

\section{Introduction}

QoS provisioning on the Internet has become an active research area over the past decade. In spite of extensive research, network-layer QoS provisioning, i.e. IntServ and DiffServ, appears unlikely to happen in the near future because of system complexity and various other reasons. Because of this, the research focus during the past two years has been turned to the overlay QoS provisioning at the application-level. Since the Internet continues to provide only the basic best-effort service, it is crucial that the overlay services adapt themselves to network congestion in order to maximize the quality perceived by end users. To facilitate the adaptation decisions made at the end hosts, these hosts have to understand the network congestion status. Bandwidth estimation plays a very important role in contributing to the understanding of the network characteristics and enhances service provisioning for QoS demand- ing applications on the Internet.

Bandwidth estimation involves two related but different bandwidth metrics: capacity and available bandwidth (avail-bw). The path capacity is a static metric if the path is fixed and known. Available bandwidth is largely influenced by the cross traffic along the path and eventually the congestion control protocol they use; therefore, it can be highly dynamic because the cross traffic can have widely different characteristics. The major challenge in the measurement of available bandwidth, is to capture the influence of the cross traffic. In the context of overlay adaptation, the network congestion is directly indicated by the available bandwidth in the network. Relatively accurate tools for capacity measurement have been proposed in [1], [2], etc, our focus is the estimation of the cross traffic intensity with the assumption that the service capacity is known.

Various avail-bw estimation techniques have been proposed in the past few years. They can be classified into two categories, self-congestion [3], [4], and model-based [5]. Despite good characteristics, such as accuracy, simplicity, speed, robustness, etc., claimed by proponents of the self-congestion approach, the congestion introduced by the probing stream inevitably changes the loading along the path in such a way that TCP flows along the path might be interfered with. Therefore, non-intrusive measurement can hardly be guaranteed. Even if temporary congestion is acceptable, the use of too many parallel measurements in a large scale overlay network using the same self-congestion based tool might collapse the network performance. Conversely, in the model-based approach, the tool is operated when the path is not congested. This brings forth the possibility of a truly non-intrusive avail-bw estimation technique. Surprisingly, little research has been reported in regard to this approach, and we argue that researchers should pay more attention and put more effort in this direction.

Currently, the most popular measurement technique is the packet-pair based method. The basic idea of packet pairs is that the sender sends a pair of packets into the net- 
work. By measuring the changes in the packet spacing, essentially the delay performance encountered by probing packets, the sender or the receiver can estimate the bandwidth properties of the network path. In the literature about the packet-pair measurement, researchers have proposed a number of techniques. However, most of these are based on some observations without deep understanding of the system, such as the one way delay increases of successive periodic probing packets when the probing rate is higher than the available bandwidth [3]. Some measurement techniques are based on some analysis which include quite strong assumptions or they are only valid for some scenarios, i.e., when the cross traffic is a constant flow [4].

The model-based measurement most related to our work is [5], in which the network characteristics were proposed to be inferred via moment-based estimators on the loss process of an active probing stream. The inference (queueing) models were developed for a single node case based on the single bottleneck link assumption. Two models were studied, $M+M / M / 1 / K$ and $M+M / D / 1 / K$, to simultaneously estimate the background traffic intensity and the buffer size of a the bottleneck link given the knowledge of the bottleneck capacity. We believe that it is questionable that the proposed performance metrics used in [5] would perform well in the measurement practice. For example, considering the end-to-end measurement situation, the packet loss might not be easily observable during the measurement period and packets might be dropped due to active buffer management instead of buffer overflow. As a more observable metric, the inter-arrival time between two packets at the receiver have not yet been addressed. This fact motivated us to conduct research on network inference via an analysis of the inter-arrival time of two consecutive probing packets at the receiver.

In the measurement practice, two types of probing sequences are commonly used: a Poisson probe sequence [6] and a periodic probe sequence. A Poisson probe can provide unbiased near continuous detection. Because the spacing between packets is essentially disturbed by the cross traffic, we propose the use of an $M_{1}+M_{2} / G I_{i} / 1$ queueing model [7] and a heavy traffic approximation [8] to capture the impact of the cross traffic on an active Poisson-type probing stream. The departure process of the probing stream is analyzed in a single bottleneck link. Our work benefited from the recent advance achieved by researchers in regard to the analysis of the departure process in queueing research and measurement practice.

The rest of the paper is organized as follows. In Section 2 we formulate the active probing problem into a queueing analysis problem of the departure process of the probing stream in an $M_{1}+M_{2} / G I_{i} / 1$ queueing system, and in a heavy traffic approximation model. In Section 3, the simulation-based performance evaluation of the proposed model is presented. Finally, conclusion remarks are drawn in Section 4.

\section{Problem Formulation}

In the model-based end-to-end measurement, the path is abstractly modelled as a series of tandem queues. The performance of an active probing stream from the sender to the receiver along this series of queues can be predicted given all the parameters of this queueing model. As an inverse process, the interesting parameters of this queueing model, including the path capacity and the avail-bw, can be estimated by measuring the performance encountered by this probing stream.

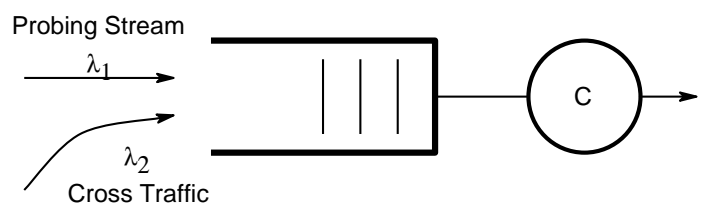

Figure 1: The available bandwidth inference model.

As the simplest starting point, it is assumed that the endto-end path characteristics are mainly determined by the bottleneck link. Therefore, the series of tandem queues can be simplified into a single queue receiving the superposition of a probing stream with arrival rate $\lambda_{1}$ and a cross traffic stream with the arrival rate $\lambda_{2}$. These are depicted in Figure 1. The aim of the proposed active measurement scheme is to infer the bandwidth information along a path using the measurement of the performance encountered by the probing packets injected into the path. The following metrics may be considered in the measurement practice: delay, delay variation, throughput and loss. The above performance metrics of the probing packets can be a function of the parameters of the end-to-end queueing model including avail-bw. Therefore, avail-bw can be inferred by plugging the measurement values into the inverse of this function. Our study indicates the potential of the path bandwidth inference scheme based on the delay variation measurement of the probing packets,

We propose to send a sequence of probing packets into the network path. Due to the impact of the cross traffic, the probing stream is "distorted" when it arrives at the receiver. The alteration of the probing arrival process can be characterized by the inter-packet departure distribution. In particular, the Squared Coefficient of Variation (SCV) is a convenient dimensionless measure to characterize the variance of this departure process. SCV is defined as the ratio of the variance of the inter departure time to the squared average inter departure time of two probing packets. By studying the departure process of the probing sequence, the interference of the cross traffic is estimated. 
This interference is mainly determined by two factors: the packet size distribution of the cross traffic and the cross traffic load. The packet size distribution can be measurable in the Internet [9], [10], etc. With the knowledge of the packet size characteristics of the cross traffic, the load of the cross traffic can be eventually estimated from the queueing model.

\subsection{The $M_{1}+M_{2} / G I_{i} / 1$ Queue}

The system in Figure 1 is modelled as a single server, infinite capacity queue operating in continuous time, which accepts two classes of customer arrivals, the probing packet arrivals and the cross traffic packet arrivals. The probing packet arrival process is assumed to be general independent and probing packets are referred as customers of class 1 . Its arrival rate is denoted as $\lambda_{1}$ and the service rate is $\mu_{1}$. The cross-traffic packets are referred as customers of class 2 and its arrival rate is denoted as $\lambda_{2}$ and the service rate is $\mu_{2}$. The probing stream is under control and provide design flexibility while the assumption of the queuing discipline and the statistical properties of cross traffic are required to be known for the construction of the queueing model.

Assume that the queueing system is steady; therefore, the traffic intensity $\rho=\rho_{1}+\rho_{2}=\frac{\lambda_{1}}{\mu_{1}}+\frac{\lambda_{2}}{\mu_{2}}<1$. Let $\Phi_{i}(s)(i=1,2)$ be the Laplace-Stieltjes transform (LST) of the service time distribution of class $i$ and $\eta_{i}(s)$ be the LST of the distribution of a busy cycle consisting solely of class $i$ customers. Denote the $n$-th probing packet arrival as $C_{n}$ of the class-1 customer arrival. Its service time is $X_{n}$. Let $B_{n}^{k}$ be the $k$-th cross traffic packet arrivals between $n$ th probing packet and $(n+1)$-st probing packet arrival of the class- 2 customer arrival. Let $I_{n}$ denote the inter-arrival time (i.a.t.) between the $n$-th and $(n+1)$-st probing packets and $D_{n}$ the corresponding inter-departure time (i.d.t.). The delay variation is defined as $J_{n}=D_{n}-I_{n}$. Note that there are possible negative jitters as well as positive jitters. Negative variation corresponds to a clustering of packets which can result in buffer overflow; positive variation corresponds to a dispersion of packets which can result in excessive delay. Because $I_{n}$ can be known in advance (the customers of class 1 are probing packets under the control), the task of characterizing $J_{n}$ can be reduced to find the property of $D_{n}$. With different of the probing strategies, the queueing models ought to be constructed correspondingly. In this paper, we focus on the analysis of the perclass departure process of a Poisson probing stream based on the $M_{1}+M_{2} / G I_{i} / 1$ queue which was originally reported in [7].

The derivation details of the Laplace-Stieltjes transform of $D_{n}$ can be further referred to [7]. Basically, $\Phi_{D_{n}}(s)$ is a function of $\lambda_{1}, \lambda_{2}, \mu_{1}, \mu_{2}, \Phi_{1}(s), \Phi_{2}(s)$. The parameters, $\mu_{1}, \mu_{2}, \Phi_{1}(s), \Phi_{2}(s)$, are essentially determined by the link capacity and the packet length statistics. The class1 stream is the probing stream under control; therefore, $\lambda_{1}$ and the class-1 packet sizes are known. If the packet length statistics of the cross traffic can be also known, $\Phi_{D_{n}}(s)$ is determined by $\lambda_{2}$ and the link capacity. Assume the average packet of cross traffic is $\overline{L_{2}}$ and the available bandwidth can be calculated as the server capacity minus $\lambda_{2} * \overline{L_{2}}$. If the statistics of $D_{n}$ can be measured via the packet-pair probing method, it is possible to infer $\lambda_{2}$ and the server capacity; eventually, the available bandwidth can be estimated.

Because the mean inter-departure time equals the mean inter-arrival time due to the flow conservation law when $\rho<1$, in the actual measurement practice, if the average inter-arrival time of probing packets is kept constant during the probing period, only the second moment or higher order moments of the inter-departure may indicate the network congestion status. Currently, we focus on the variance of the inter-departure time of $D_{n}$. The variance of $D_{n}$ can be obtained by differentiating $\Phi_{D_{n}}(s)$ twice and evaluating at $s=0$. Therefore, by measuring this variance, the traffic intensity of the cross traffic can be inferred.

\subsection{Fischer-Stanford's approximation}

In this section we study an $M+G I / G I_{i} / 1$ queue in a heavy traffic, which receives the superposition of a Poisson probing stream with the arrival rate $\lambda_{1}$ and a general-type cross traffic stream with the rate $\lambda_{2}$. These two streams have the service rate $\mu_{1}$ and $\mu_{2}$ respectively. Let $\rho_{1}=\frac{\lambda_{1}}{\mu_{1}}$ and $\rho_{2}=\frac{\lambda_{2}}{\mu_{2}}$. $Y_{j}$ is the packet service time of cross traffic. In [8] Fischer and Stanford derived the approximations for the per-class interdeparture time for a $\sum_{i} G I_{i} / G I_{i} / 1$ queue.

Let $S C V_{I_{n}}$ be the SCV of the interarrival time and $S C V_{D_{n}}$ be the SCV of the interdeparture time of the probing stream, and let $\rho$ be the overall loading of the queue and $\rho=\rho_{1}+\rho_{2}$. Following Fischer-Stanford's approximations in Eqn. (25) in [8], the following Eqn. 1 holds.

$$
S C V_{D_{n}}=\left(1-\rho^{2}\right) S C V_{I_{n}}+\rho^{2} S C V_{D_{n, 1}}
$$

where $S C V_{D_{n, 1}}$ is the SCV of the interdeparture time of the probing stream for $\rho \rightarrow 1$.

After some algebraic manipulations, we have

$$
S C V_{D_{n, 1}}=\frac{\lambda_{2} \mu_{1}^{2}\left(\lambda_{2}+\lambda_{1}\left(1+\operatorname{Var}\left[Y_{j}\right] \mu_{2}^{2}\right)\right)}{\left(\lambda_{2} \mu_{1}+\lambda_{1} \mu_{2}\right)^{2}} .
$$

For the Poisson probing stream in the $M+G I / G I_{i} / 1$ queue, $S C V_{I_{n}}=1$ and Eqn. 1 can be simplified into Eqn. 3.

$$
S C V_{D_{n}}=1-\rho^{2}+\rho^{2} S C V_{D_{n, 1}}
$$




\subsection{Moment matching of the packet size distribu- tion of cross traffic}

Compared with the $M_{1}+M_{2} / G I_{i} / 1$ queueing model, Fischer-Stanford's approximation only requires the first and second moment of the packet size distribution of the cross traffic. However, the complete packet size distribution is needed to solve the $M_{1}+M_{2} / G I_{i} / 1$ queuing model. With the tremendous efforts in Internet measurement [9], [10], etc, it is fair to assume that we can know the first and second moments of the packet size distribution of the Internet traffic. We propose to map these two moments information of packet size distribution of the cross traffic into a generalized distribution; then, use the $M_{1}+M_{2} / G I_{i} / 1$ queueing model to infer the cross traffic intensity.

Assume that we know the first and second order information of the packet size distribution of Internet traffic. A general packet size distribution can be created with a certain mean value $\mu$ and SCV $c$ by linking two phases [11].

- Case I $(c=0)$ : only one phase with a constant distribution (mean value $\mu$ ).

- Case II $(0<c<1)$ : serial switching of a constant distribution with the mean value $m_{1}=\mu \cdot c$ and a negative-exponential distribution with the mean value $m_{2}=\mu \cdot(1-c)$.

- Case III $(c=1)$ : only one phase with a negativeexponential distribution with the mean value $\mu$.

- Case IV $(c>1)$ : parallel switching of two phases with a negative-exponential distribution and the parameters $m_{1 / 2}=\frac{\mu}{1 \pm \sqrt{\left(c^{2}-1\right) /\left(c^{2}+1\right)}}$ (mean value) and $p_{1 / 2}=\frac{1}{2} \cdot\left(1 \pm \sqrt{\left(c^{2}-1\right) /\left(c^{2}+1\right)}\right)$ (branching probabilities). This corresponds to a hyperexponential distribution of second order, its parameters meet the symmetrical condition $p_{1} \cdot m_{1}=p_{2} \cdot m_{2}$.

With the above mapping scheme, the mean and variance of the mapped packet size distribution is the same as the given first and second order information of the packet size distribution of Internet traffic.

\section{Performance Evaluation}

We conducted intensive simulation experiments to study the accuracy of the proposed model. The whole simulation model was implemented using SimLib 2.2 [12]. In this section, we discuss the accuracies of the Poisson probing using the $M_{1}+M_{2} / G I_{i} / 1$ queue and Fischer-Stanford's approximation to infer the cross traffic load. The Relative Estimation Error is defined as the ratio between the estimated load error and the real cross traffic load in terms of percent.

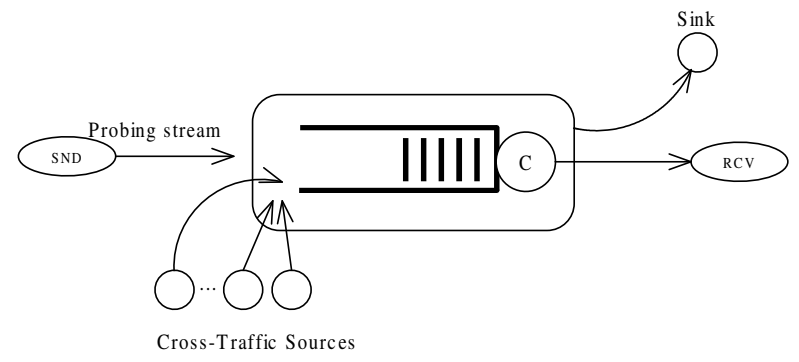

Figure 2: A single bottleneck topology.

\subsection{Network Configuration}

Shown in Figure 2 is a single server queue which accepts the superposition of a probing stream and an aggregate cross traffic stream. The link capacity is $2 \mathrm{Mbps}$ and the buffer size is 500 Kbytes. There are ten homogeneous cross traffic sources, and three types of packet size distributions of the cross traffic are considered. One is that cross traffic consists of three types of packet sizes of $\{40,550,1500\}$ bytes with a distribution of $\{40 \%, 50 \%$, $10 \%\}$. These parameters were also selected in the simulation study in [3]. The second type of cross traffic is that the packet size is distributed with the truncated negative exponential distribution with the mean 441 bytes and the Maximum Segment Size 1500 bytes. The third type of cross traffic is that the packet size is distributed with the negative exponential distribution with the mean 441 bytes. By changing the arrival rate of the cross traffic streams, the loading of the cross traffic can be configured. To have a minimum probing load, the packet size of the probing stream is configured as the minimum packet size 40 bytes unless this size is explicitly stated.

\subsection{Departure process of the probing stream}

For the measurement purpose, we were interested in investigating the first and second order characteristics of the departure process of the probing stream for different type of arrival processes of cross traffic. For the first order statistics, the gap ratio is introduced as the ratio between the average inter departure time and the average inter arrival time of two probing packets at the bottleneck queue. Because the probing packets might be dropped due to lacking of buffer, we are only interested in the gap information of two consecutive probing packets, which is denoted the conditional gap ratio. To this end, each packet is assigned with a unique sequence number. When the path load is far less than 1 , packet dropping is very small. Based on the flow conservation property, all the probing packets leave the network eventually and, hence, the average output gap equals the average input gap regardless of the types of arrival processes of the cross traffic and traffic intensity. Therefore, this first order characteristics of the departure 
process cannot provide information about the exact loading of the cross traffic. Therefore, we turned to investigate the second order characteristics of the departure process of the probing stream.

Figure 3 depicts the second-order property of the departure process of the probing stream via the simulation. Like the conditional gap ratio, we were interested in the conditional SCV of two consecutive probing packets received at the receiver. With the different types of arrival process of the cross traffic and traffic intensity, as shown in Figure 3, the SCV increases when the normalized probing load increases. For different types of the arrival process of the cross traffic, the probing stream has a similar performance; therefore, the analysis of the per-class departure process for the Poisson-type cross traffic can be used to infer the non-Poisson type cross traffic without much accuracy loss.

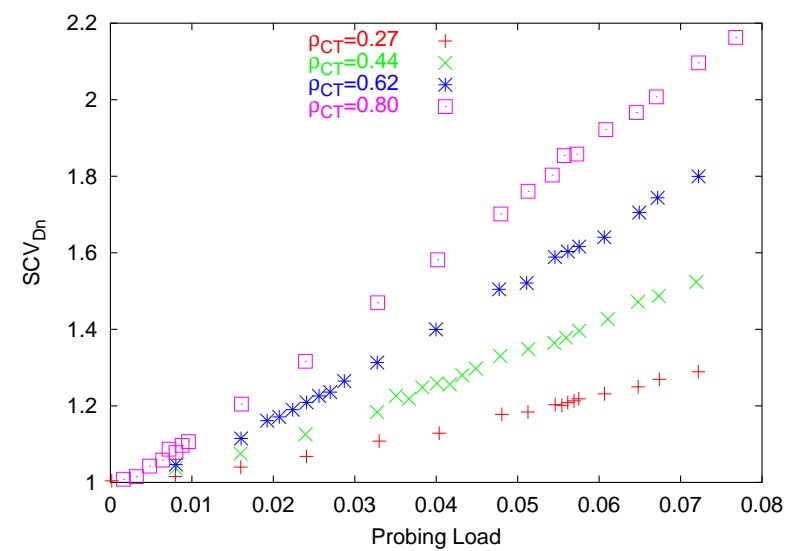

Figure 4: The relationship between conditional $S C V_{D_{n}}$ and the Poisson probing load via simulation with Poisson arrival process of cross traffic and the packet size is distributed with the negative exponential distribution with the mean 441 bytes and the probing packet size is 40 bytes.

In Figure 4 it is shown that the performance of the departure process of the Poisson probing stream is largely impacted by the loading of the cross traffic. From these findings that provide the base, the intensity of the cross traffic can be inferred by measuring the performance metrics of the departure process of the probing stream, i.e., the delay variance. Given the knowledge of the capacity information, the available bandwidth is determined by the difference between the capacity and the cross traffic intensity.

\subsection{Model Validation}

Plotted in Figure 5 are the SCVs of the probing stream obtained during the simulation for different probing loads, together with the results obtained using the analysis of the departure process in the $M_{1}+M_{2} / G I_{i} / 1$ queue and
Fischer-Stanford's approximation. It is shown in Figure 5(a) that the delay variance of the probing stream can be accurately predicated by solving the $M_{1}+M_{2} / G I_{i} / 1$ queue model. This provide the base that the cross traffic intensity can be inferred accurately. In Figure 5(b), the accuracy using Fischer-Stanford's approximation is not as good as that using the $M_{1}+M_{2} / G I_{i} / 1$ queuing model. In addition, the accuracy using Fischer-Stanford's approximation when the path load is heavy, is better than that when the load is light. This is due to the fact that Fischer-Stanford's approximation is essentially a heavy traffic approximation and it is more accurate when the path is more heavily loaded. Fischer-Stanford's approximation only requires the knowledge of the first and second order statistics of the packet size distribution of the cross traffic; however, the packet size distribution is needed to solve the $M_{1}+M_{2} / G I_{i} / 1$ queuing model. The inaccurate knowledge of the packet size distribution of cross traffic may introduce inaccuracy of the cross traffic estimation.

\subsection{Performance of moment matching of the packet size distribution of cross traffic}

Based on the Internet measurement, assume that the Internet traffic consist of three types of packet sizes of $\{40$, $550,1500\}$ bytes with a distribution of $\{40 \%, 50 \%, 10 \%\}$, which is denoted as typical CT in Figure 6. In what follows, we illustrate a simple first order mapping scheme for the packet size distribution using the negative exponential distribution for the comparison purpose with the two moment matching proposed in section 2.3. Because there is only one parameter in exponential distribution, we conducted the first moment mapping so that the average packet service time in the mapped negative exponential distribution is the same as that in the typical CT. In Figure 6(a), the predicted SCV curves using the $M_{1}+M_{2} / G I_{i} / 1$ queueing model for the typical CT and the NegExp CT are quite close in all the four loading scenarios. After a careful examination, we found that the SCV of the packet size of this typical CT is $S C V_{\text {typical }}=0.96$, which is quite close to the SCV of the negative exponential packet size distribution $S C V_{\text {NegExp }}=1$.

In Figure 6(b), the performance of the two moment matching of packet size distribution of the cross traffic is depicted. It can be shown that the accuracy of the SCV curves is more improved using the two moment matching scheme than that using the first moment only matching scheme.

\subsection{Accuracy of the avail-bw inference 3.5.1 The $M_{1}+M_{2} / G I_{i} / 1$ Queue}

In Figure 7, the accuracy of the intensity inference of the cross traffic using the $M_{1}+M_{2} / G I_{i} / 1$ queue is depicted under a typical cross traffic. The estimation accuracy improves with the normalized probing load increasing. When 


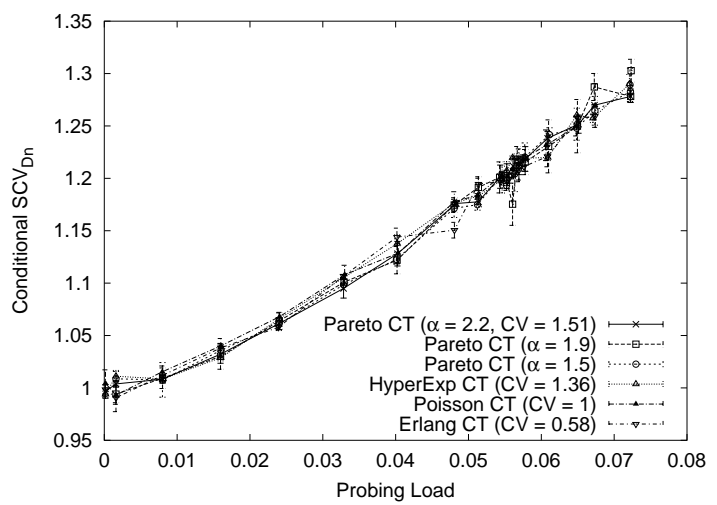

(a) $\rho_{C T}=0.27$

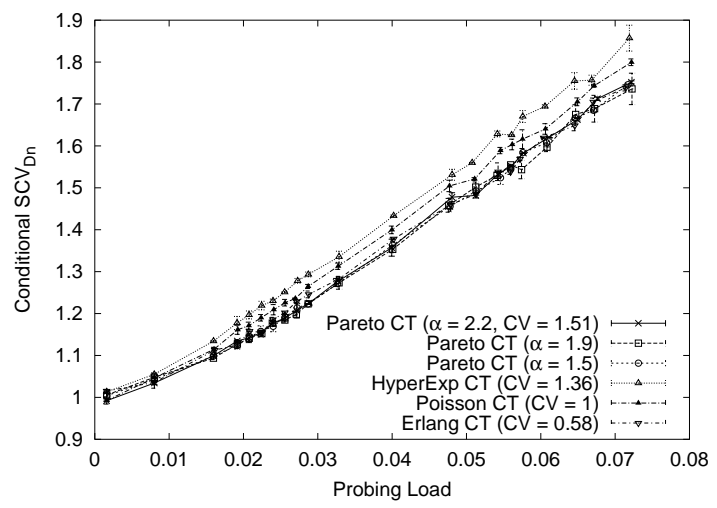

(b) $\rho_{C T}=0.62$

Figure 3: Relationship between the conditional $S C V_{D_{n}}$ and the Poisson probing load via simulation with various type cross traffic arrival processes and the packet size is distributed with the negative exponential distribution with the mean 441 bytes and the probing packet size is 40 bytes.

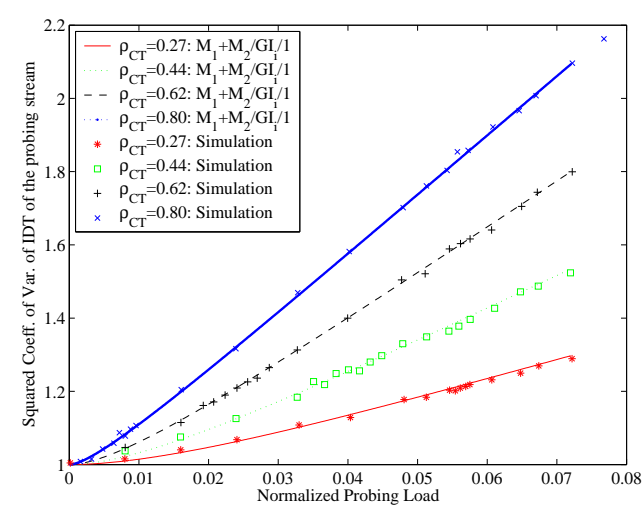

(a) $M_{1}+M_{2} / G I_{i} / 1$

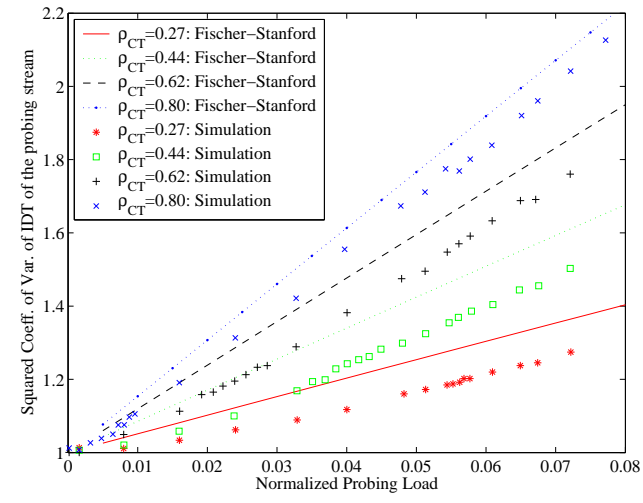

(b) Fischer-Stanford's Approximation

Figure 5: The conditional SCV of inter-departure time of the Poisson probing stream via simulation when the packet size is distributed with the negative exponential distribution with the mean 441 bytes and the probing size is 40 bytes.

the normalized probing load is larger than 0.05 , the relative estimation error is less than $5 \%$. For other two types of cross traffic, similar estimation accuracies were achieved.

\subsubsection{Fischer-Stanford's approximation of the $M_{1}+$ $M_{2} / G I_{i} / 1$ queue}

In Figure 8, the accuracy of the intensity inference of the cross traffic is depicted for Poisson probing with a probing packet size of 40 bytes using Fischer-Stanford's approximation. The estimation accuracy improves with the normalized probing load increasing. However, even when the probing load increases further, the estimation error decreases very slow. When the normalized probing load reaches 0.07 , the relative error is quite high up to $30 \%$. Note that the accuracy when the cross traffic load is high is better than that when the cross traffic load is low. This is because Fischer-Stanford's approximation is essentially a heavy traffic approximation.

\section{Conclusion}

In this paper we propose to probe the end-to-end path to estimate the avail-bw using small packet size with the negative exponential inter-leaving time between consecutive probing packets. The small packet size helps to have the minimum impact upon the network by the probing stream. By studying the departure process of the probing stream, the interference of the cross traffic is estimated and, hence, 


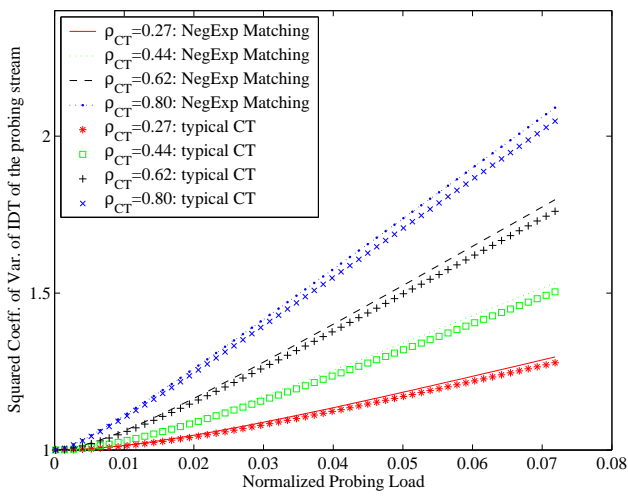

(a) First moment matching using negative exponential distribution

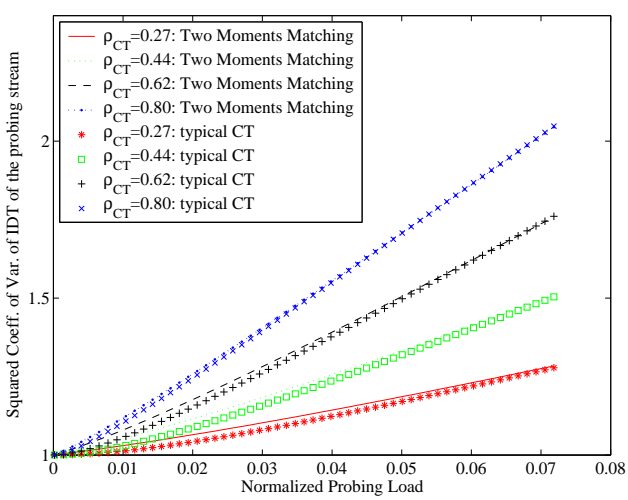

(b) Two moments matching

Figure 6: Performance of the moment matching of packet size distribution of the cross traffic using the $M_{1}+M_{2} / G I_{i} / 1$ queueing model.

the cross traffic intensity can be inferred. Our proposed probing scheme works when the path is not congested. This brings forth the possibility of a truly non-intrusive avail-bw estimation technique.

The accuracy of the estimation of the cross traffic are discussed for Poisson probing. The accuracy using the $M_{1}+M_{2} / G I_{i} / 1$ queue is very good. For three types of the cross traffic, the estimation accuracy improves with increasing probing load. When the normalized probing load is larger than 0.05 , the relative estimation error is less than $5 \%$ for the four loading scenarios. However, it turns out that the knowledge of the packet size distribution have impact upon the accuracy of the inference process.

For the Poisson probing, the estimation accuracy using Fischer-Stanford's approximation is not as good as that using the $M_{1}+M_{2} / G I_{i} / 1$ queue. Naturally, the accuracy of the estimation improves with increasing probing load. However, even when the probing load increases further, the estimation error decreases very slow. When probing packet size is 40 bytes and the normalized probing load reaches 0.07 , the relative error is still quite high up to $30 \%$. The accuracy when the cross traffic load is high is better than that when the cross traffic load is low.

We are currently evaluating the proposed model-based avail-bw measurement in a testbed and along real Internet paths with the currently available avail-bw measurement tools. In this paper a single bottleneck is assumed along one end-to-end path. For multiple congested links, the exact queue analysis is very hard; therefore, we are more interested in investigating the approximated analysis of the departure process of this tandem queues.

\section{References}

[1] C. Dovrolis, P. Ramanathan, and D. Moore, "What do packet dispersion techniques measure?" in Proceedings of INFOCOM, vol. 2, Piscataway, NJ, USA, 2001, pp. 905-14.

[2] K. Lai and M. Baker, "Nettimer: a tool for measuring bottleneck link bandwidth," in Proceedings of $3 \mathrm{rd}$ USENIX Symposium on Internet Technologies and Systems. (USITS'01), Berkeley, CA, USA, 2001, pp. $123-34$.

[3] M. Jain and C. Dovrolis, "End-to-end available bandwidth: Measurement methodology, dynamics, and relation with TCP throughput," in Proceeding of ACM SIGCOMM, 2002.

[4] N. Hu and P. Steenkiste, "Evaluation and characterization of available bandwidth probing techniques," IEEE JSAC Special Issue in Internet and WWW Measurement, Mapping, and Modeling, vol. 21, no. 6, pp. 879-893, Aug. 2003.

[5] S. Alouf, P. Nain, and D. Towsley, "Inferring network characteristics via moment-based estimators," in Proceedings of INFOCOM, vol. 2, no. 1045-54, Piscataway, NJ, USA, 2001.

[6] V. Paxson, G. Almes, J. Mahdavi, and M. Mathis, "RFC 2330: Framework for IP performance metrics," May 1998, status: INFORMATIONAL. [Online]. Available: ftp://ftp.internic.net/rfc/rfc2330.txt,ftp:// ftp.math.utah.edu/pub/rfc/rfc2330.txt 


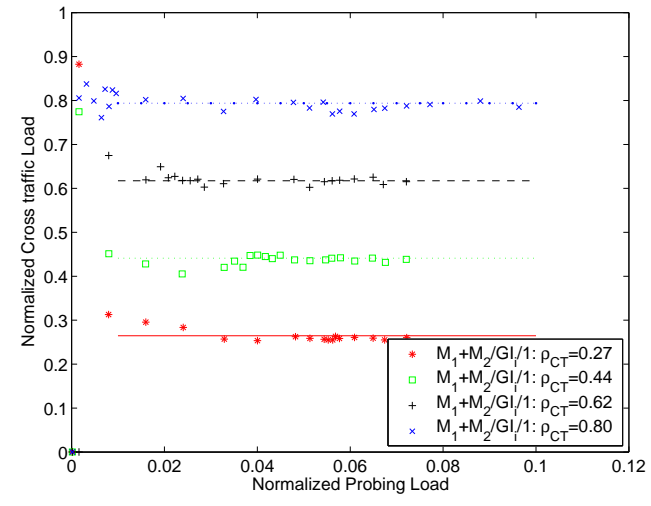

(a) Cross Traffic Estimation

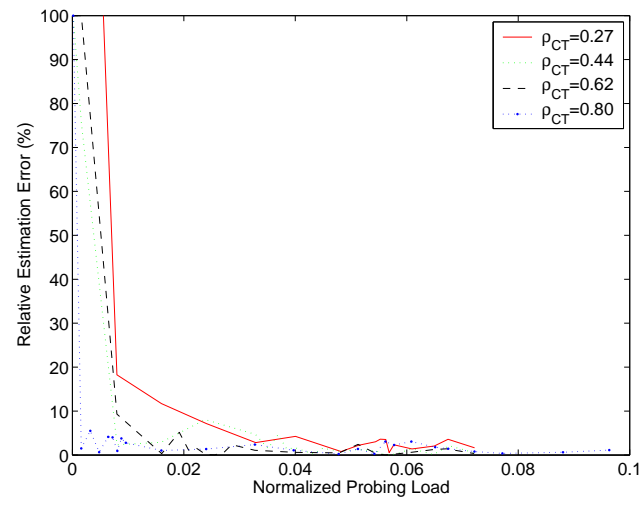

(b) Relative Estimation Error

Figure 7: Accuracy of the Poisson probing using $M_{1}+M_{2} / G I_{i} / 1$ when the packet size is distributed with three types of packets $\{40,550,1500\}$ bytes and the probability distribution $\{40 \%, 50 \%, 10 \%\}$ and the probing packet size is 40 bytes.

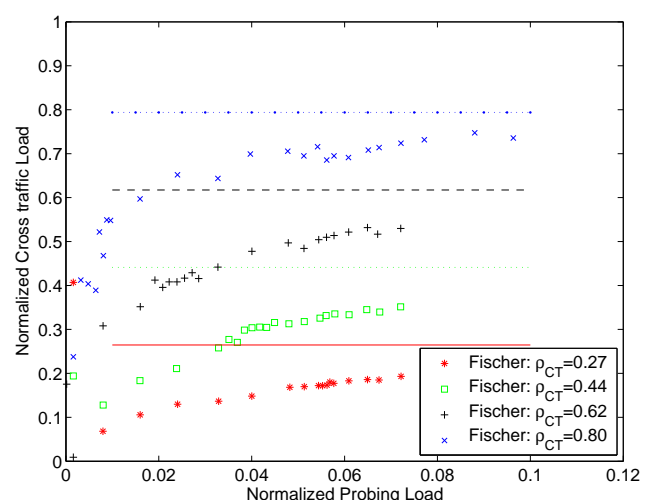

(a) Cross Traffic Estimation

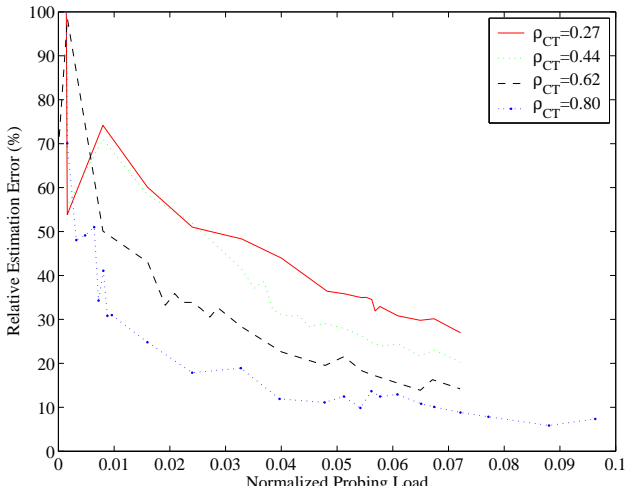

(b) Relative Estimation Error

Figure 8: Accuracy of the Poisson probing using Fischer-Stanford's approximation when the packet size is distributed with the negative exponential distribution with the mean 441 bytes and the probing packet size is 40 bytes.

[7] D. Stanford and W. Fischer, "The interdeparturetime distribution for each class in the $\sum_{i} M_{i} / G_{i} / 1$ queue," Queueing Systems, vol. 4, no. 3, pp. 179-91, July 1989.

[8] W. Fischer and D. Stanford, "Approximations for the per-class waiting time and interdeparture time in the $\sum_{i} G I_{i} / G_{i} / 1$ queue," Performance Evaluation, vol. 14, no. 2, pp. 65-78, 1992.

[9] V. Paxson and S. Floyd, "Wide area traffic: the failure of poisson modeling," IEEE/ACM Transactions on Networking (TON), vol. 3, no. 3, pp. 226-244, 1995.
[10] S. McCreary and K. Claffy, "Trends in wide area IP traffic patterns - a view from ames internet exchange," in ITC Specialist Seminar, vol. 2, Sep 2000. [Online]. Available: http://www.caida.org/ outreach/papers/2000/AIX0005/AIX0005.pdf

[11] S. Bodamer and K. Dolzer, Introduction to the Concepts of the C++ Simulation Library, version 2.1, IND, University of Stuttgart, 2001. [Online]. Available: http://www.ind.uni-stuttgart.de/INDSimLib/

[12] IND. University of Stuttgart, SimLib library 2.2. [Online]. Available: http://www.ind.uni-stuttgart.de/ INDSimLib/ 